\title{
How GRID could improve E-Learning in the environmental science domain
}

\author{
Stefan Wesner, Konrad Wulf \\ High Performance Computing Centre Stuttgart (HLRS), Allmandring 30, 70550 Stuttgart \\ \{wesner,wulf\}@hlrs.de \\ Mark Müller \\ ILPOE Universtiy of Stuttgart, Keplerstrasse 11, 70174 Stuttgart \\ mamue@ilpoe.uni-stuttgart.de
}

\begin{abstract}
This paper will outline the requirements for an interactive e-learning system defined as part of the German research project GIMOLUS [1]. After a short overview over the Open Grid Service Architecture (OGSA) it will be shown that the capabilities of existing e-learning solutions are too limited in order to fulfil these requirements. The last part will show how a GIMOLUS system could be built using a GRID service architecture and what the benefits are in doing so.
\end{abstract}

Keywords: Interactive E-Learning, GRID, Web Services, GIS

\section{INTRODUCTION}

Within the last years a wide range of E-Learning systems have been brought to market. These systems have become more and more complex and each of them covers a wide range of functionality. Many systems assist the user throughout the entire process of producing and publishing learning material over the web. It is certainly desirable when a system that perfectly matches your needs already exists. But very often such a perfect system would only exist if one could take pieces of existing systems and rearrange them to a new system. Ideally you could even add your own components to this newly generated system.

This means that an E-Learning system should be build as a distributed application, deployed on several hosts. These distributed applications would need an underlying infrastructure that allows each of these components to access and share resources or functionality across the Internet. Such problems have already been addressed in the area of scientific distributed computing and lead to the definition of a concept that is often referred to as GRID technology [2].

In this working paper we are going to analyse how GRID technology can be used to improve the architecture and capabilities of an E-Learning system that mainly utilizes a geo information system (GIS).

\section{THE GIMOLUS PROJECT}

\subsection{PROJECT OVERVIEW}

The overall task of the project is the development of learning modules for different disciplines in environmental science with a focus on applying models and geo-information systems (GIS). GIMOLUS modules are aiming to enrich existing teaching units with interactive exercises.

The framework for integrating, connecting and arranging the learning modules consists of a module server with a basic graphical user interface and a shared virtual landscape. The Internet based combination of models and GIS is allowing the student to change model parameters and instantly see the effects on the underlying spatial dataset. Exercises are meant to have a case study character.

One benefit of the computer based infrastructure is that the students can experience the models rather than only hear, read and think about them in lectures and books. 
The main objective of the modules is to provide an interactive view on the model and the landscape. Being supplied all the necessary information and feedback, the student can actively manipulate the models and gets immediate responses to his actions. Thus, the student can acquire a deeper understanding of the models and how changes in parameters and in the algorithms influence them. The virtual landscape's elements have been modified in order to fit better to the teaching main aspects of landscape ecology. Recognizing the same landscape areas in different learning modules makes it possible to understand and compare different scientific disciplines, experiencing each from their own point of view. Students are thus encouraged to construct or deepen their very own conceptual view on the network of the involved natural sciences. Combining didactically well-prepared learning contents, enhanced through the exemplification effect with a virtual landscape, is the GIMOLUS approach to increase the students' level of spatial, environmental and multidisciplinary thinking.

The project is focused on methodology and content production and not on technology and software development. Concerning technology, the idea was to rely on existing E-Learning tools and integrate them for developing and delivering the interactive exercises. The high technical potential of the Internet with respect to networking and personal exchange will be used in a didactically well-founded manner to achieve a high degree of learning efficiency for the participating students.

\subsection{GIMOLUS Platform}

The platform is based on a GIS, a database, client and server side scripts. The content is divided into so-called learning modules, representing different topics. The linking element between all these modules is the underlying virtual landscape, which is based upon existing datasets from a real landscape in southwest Germany.

The GIMOLUS module server is technically based on a combination of XML data modules and XSL-transformations. The graphical user interface will be a set of dynamically generated web pages, functionally reduced to user adaptive navigation within the content. Student communication with lecturers and tutors will be realised via e-mail and module specific discussion forums using the standalone groupware system BSCW that will be adapted to the needs of GIMOLUS.

The GIS components used in GIMOLUS are provided by the ESRI [4]. The two GIS servers are split into an ArcIMS-server (ArclnternetMapserver, [4]) and a server with an ArcGIS system used via Metaframe XP (by CITRIX [4]). The geometries and attribute data of the landscape are being administered by ArcSDE [4], which in turn makes use of a standard database, in this case MS SQL Server ([8]).

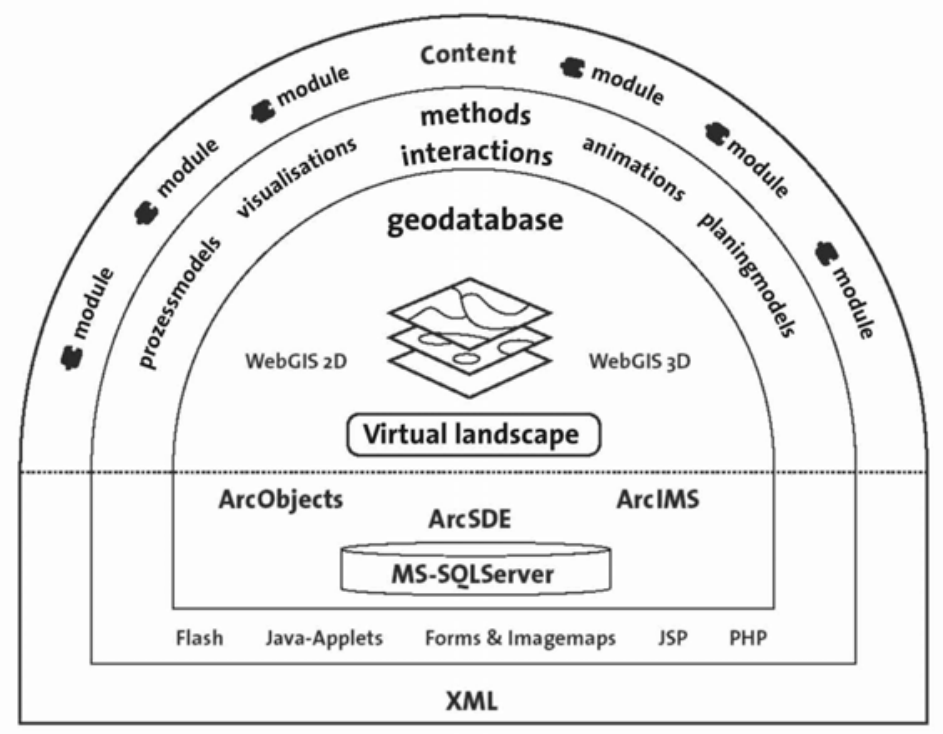


FIGURE 1: The Platform of GIMOLUS

\subsection{Example Scenario}

In order to give a better impression on the type of E-Learning system GIMOLUS is implementing an example scenario is described here. The example exercise is the calculation of soil erosion according to the universal soil loss equation (USLE) and its spatial distribution in a given region of the virtual landscape. After a basic introduction to soil erosion, the first part of the training exercise will aim at deepening the understanding of spatial correlations and patterns which need to be considered regarding erosion on a landscape scale.

Another task is the reduction of erosion by manipulating structural elements and land use in a certain parts of the investigated region.

The manipulation of data and observation of the model's behaviour is both done within ArcGIS. The next steps in the exercise will become more and more complex by supplying either more changeable parameters or by enriching the task as a case study with more interrelations and restrictions like for example monetary aspects.

The user has to use several GIS standard tools like queries and statistics to visualise and analyse the results. It is also possible to view certain parts of the data three dimensionally for the sake of analysing structural and spatial correlations within the data. The last step will be the interpretation and discussion of the results, which will be submitted to a tutor for marking.

\section{MISSING FEATURES OF OFF-THE-SHELF E-LEARNING SOLUTIONS}

Existing E-Learning software cannot offer all the features needed for the GIMOLUS project. Especially embedding custom tailored components like applets into learning units which have been generated and organized by a standard E-Learning system was not satisfactory because of the closed nature of the current solutions.

Ideally, from the GIMOLUS point of view, a perfect E-Learning solution would consist of highly integrable building blocks, supposedly provided by different vendors, which could easily be combined to shape unique learning units. In this sense, modules like user administration, group working, content creation, content provision and so on could be selected and replaced as needed. This would require on the one hand that each of these components exhibits and makes use of standardized interfaces. On the other hand such components would have to be based on the same underlying infrastructure that provides basic functionality such as service location, session management and allowing for dynamical addition or removal of such components. We believe that E-Learning modules implemented as GRID services could constitute such a solution.

\section{RELEVANT GRID CONCEPTS}

At the time the technical term "GRID" came to life, the focus was on the infrastructure within high performance computing (the book that gave birth to the expression in that context: [2]). Now, recent developments within the GRID domain make the concepts and ideas also valuable for other computing areas. As shown in [2] the GRID technology, once only being used for specialized niche solutions for computational science, is now becoming mainstream. One major improvement supporting this development has been made through taking advantage of standardized technologies such as XML Web Services [4].

The Open Grid Service Architecture (OGSA) defined in [3] invents the term of the "Grid Service". In OGSA, a GRID supplies virtual organizations (VO) with a set of standardized, extensible services that can be aggregated in various ways. A GRID Service has got standardized semantics and publicly exposed interfaces. The technology being used to describe these services is the Web Service Description Language (WSDL). WSDL, standardized by the World Wide Web Consortium (W3C), does not only describe service interface definitions, but also possible protocol bindings for service invocation. These bindings can be carried out using 
distributed communication protocols such as HTTP or local bindings in order to optimise communication between services on the same host.

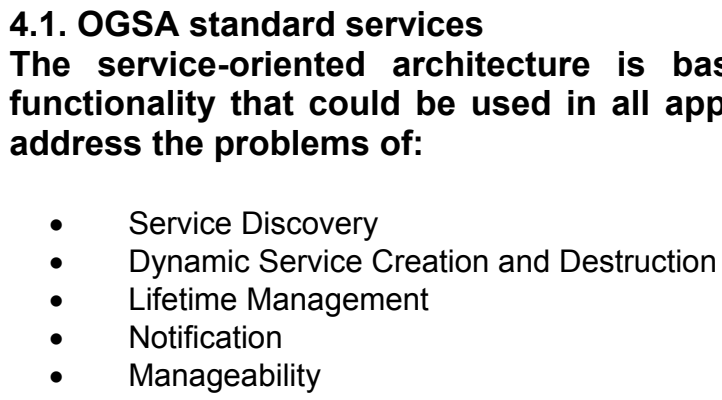

- $\quad$ Service Discovery

- Dynamic Service Creation and Destruction

- Lifetime Management

- Notification

- Manageability

\subsubsection{Service Discovery}

In a dynamic environment a mechanism for locating a service and providing information on how a connection with it can be established and handled is needed.

\subsubsection{Service Creation and Destruction}

As services are not necessarily available or needed all the time mechanism for creating and managing services together with the possibility to destroy services must be provided by a basic infrastructure.

\subsubsection{Lifetime Management}

OGSA defines interfaces for managing a service's lifetime, especially, in case of failed operations, for reclaiming associated services and states. Some services depend or build on others, which also have to recover after failure. Grid services meet these requirements by sending out keep-alive messages in regular intervals as long as the connection shall be maintained.

\subsubsection{Notification}

For asynchronous message exchange among components, OGSA defines a set of interfaces for sending and receiving notifications.

\subsubsection{Manageability}

Within a production environment it is necessary to supervise the GRID services, ensuring Quality of Service (QoS) for the clients.

\section{A GRID BASED GIMOLUS SYSTEM}

In the next sections, we will focus on how a GRID service oriented E-Learning system could address the problems within GIMOLUS.

\subsection{Common infrastructure}

One of the essential problems that could not be solved by existing E-Learning software was to provide a customized client for the students enabling a common view on the different elements that make up the GIMOLUS system. This was due to the closed nature of the applications that did not allow linking the content of one system with the content stored in another.

The idea of a basic infrastructure not only essential for common services like the management of user profiles but also for defining common services and interfaces together with dynamic binding and service location. This would enable the production of dedicated clients that utilize the functionality offered by components and offer a uniform view on it.

\subsection{Interactive exercises}

The most important contents produced within the GIMOLUS project are the interactive exercises. They have been realized as traditional client server applications that require individual development of the clients. As the elements of these training exercises were often based on common building blocks it would be feasible to define functional components e.g. for solving a linear equation system as a service component that could be used by all exercises. 
Preferably, an exercise should be composed out of a set of standardized input methods and functional components. Such an environment would allow the E-Learning content provider to concentrate on the topic rather than on technology. Beside this the availability of standardized services such as a GIS simulation component with different underlying landscapes would offer the student a wider range of possible scenarios. Another issue is that a GRID based GIS simulation module would also benefit from scalability features as new GIS components could be added as needed.

\subsection{Resource Location}

In the current solutions, there typically exists a single entry point, which is very often a web page that is being displayed after logging in, supplying the content that matches the profile of the authenticated users. The content that is presented to the student has been selected and organized in its structure by an author. There is for sure no alternative for this procedure if the content is meant as a supplement or replacement for a lecture at universities, which is the focus of GIMOLUS.

An interesting addition would be the possibility to include related material dynamically to the provided content.

Another scenario would be that the author of the course could select the content from different locations and compose a dedicated solution for its students using spread network resources together with his own content. In order to make this scenario possible it is necessary to classify the content in a standardized way e.g. using a metadata standard. Then this metadata should be stored together with connection specific information in a service repository.

\subsection{Dynamic Settings}

Another problem that could not be solved for GIMOLUS using existing solutions was the integration of supporting applications such as groupware and proprietarily developed software. In an architecture where a system is composed of a set of distributed services rather than one monolithic system, it would be possible to add or remove such supporting applications as needed and replace an application of a specific type with another application implementing the same desired interface.

\subsection{Possible architecture}

A GRID based E-Learning system would require to specify in analogy to the specification of the common services in OGSA a set of domain specific services that could be based in a first approach on coarse grained building blocks such as groupware, content server, GIS simulator. For a real service oriented architecture the specified services must have a higher granularity, so that the composition of the entire system can be made on a component level, rather than on an application level.

The specification process for a GRID based E-Learning environment must lead to the specification of domain specific services that could be seen as general needed components for all kind of E-Learning applications. Besides the common services provided by OGSA and the domain specific services, there is of course still the necessity of implementing application specific services if specialized functionality is needed. 


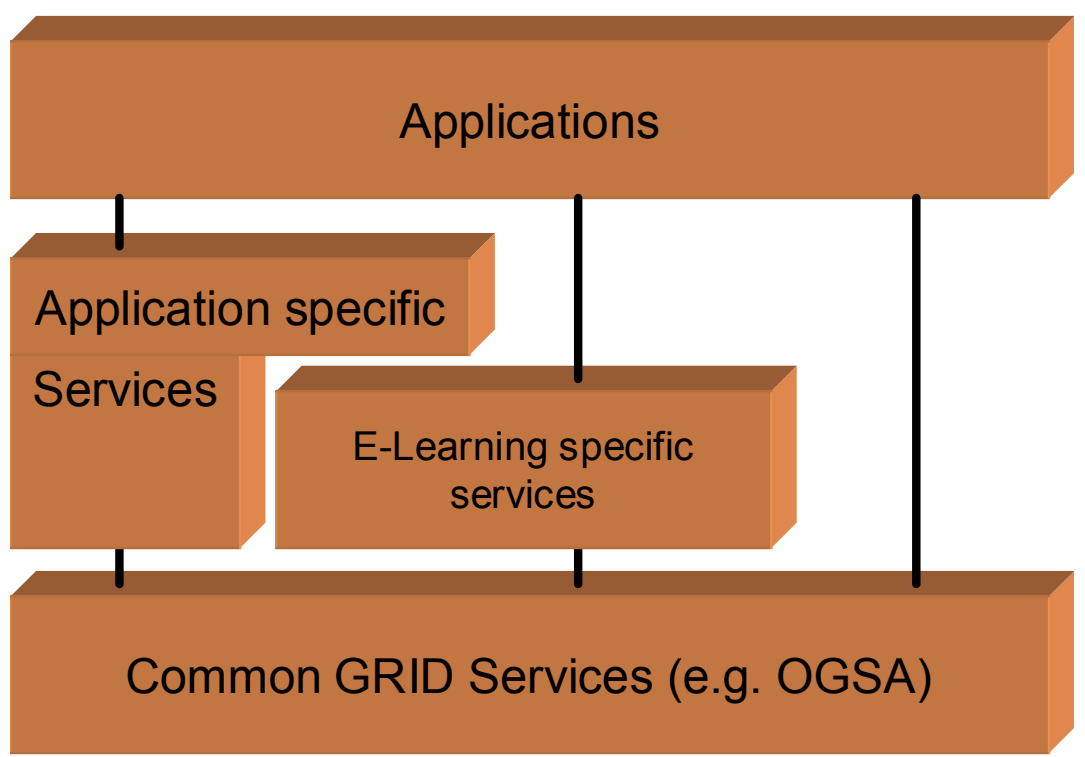

FIGURE 2: Relations of service levels

Such an architecture allows easy integration of proprietarily developed components ("application specific services") as part of the underlying infrastructure provided by the common and E-Learning specific services of a E-Learning GRID environment.

\section{CONCLUSION}

It has been shown that despite the fact that GRID technologies has been invented without having the requirements of E-Learning systems in mind it is possible to transfer the concepts and technology to the E-Learning domain.

E-Learning systems can benefit in terms of flexibility and scalability if they are built out of existing standard service components combined with specific services. Beside the technological aspect of using GRID for building E-Learning system it is possible to build completely new types of E-Learning systems if the term resource that is limited to computing resource in its original meaning is extended to cover also exercises, content and other resources in order to reach for example an European wide GRID where E-Learning resources are shared and can be accessed.

\section{ACKNOWLEDGMENTS}

This paper is partially based on the experiences gained from the German research project GIMOLUS that is funded by the BMBF .(Bundesministerium für Bildung und Forschung) within the programme 'Neue Medien in der Bildung'1 ([6])

\section{REFERENCES}

[1] GIMOLUS, Web Site http://www.gimolus.de

[2] Foster et al. (1999) Grid Services for Distributed System Integration, I. Foster and C. Kesselman, eds., The Grid: Blueprint for a New Computing Infrastructure, Morgan Kaufmann, San Francisco.

[3] Foster et al., The Physiology of the Grid: An Open Grid Services Architecture for Distributed Systems Integration, tech. report, Globus Project; http://www.globus.org/research/papers/ogsa.pdf

[4] Heather Kreger IBM Software Group, Web Services Conceptual Architecture, http://www3.ibm.com/software/solutions/webservices/pdf/WSCA.pdf

[5] ESRI - GIS and Mapping Software, http://www.esri.com

[6] BMBF Program Neue Medien in der Bildung, http://www.medien-bildung.net

[7] CITRIX - Metaframe, http://www.citrix.com

\footnotetext{
${ }^{1}$ Use of new media within education
} 
How GRID could improve E-Learning in the environmental science domain

[8] Microsoft SQL Server, http://www.microsoft.com/sql/default.asp 